\title{
Analysis of the Relation between Sun Motion Law and the Change of Earth's Environment
}

\author{
Zhe Yin, Dongxu Liu \\ Mathematics Department, Yanbian University, Yanji, China \\ Email:yinzhe@ybu.edu.cn
}

Received 29 February 2016; accepted 5 March 2016; published 8 March 2016

Copyright (C) 2016 by authors and Scientific Research Publishing Inc.

This work is licensed under the Creative Commons Attribution International License (CC BY). http://creativecommons.org/licenses/by/4.0/

c) (i) Open Access

\begin{abstract}
We deduce and obtain the law of the motion of the sun by analyzing the law of motion between the moon, the earth, the sun, and the Polaris. Then we obtain the root of the change of earth's environment from the law. Meanwhile we analyze the form of the present state of the universe movement and the conditions of energy homeostasis. It may provide reference value for related research.
\end{abstract}

\section{Keywords}

\section{Sun Motion, Earth, Environment}

\section{Introduction}

In the past few hundred years, great achievements have been made in astronomy. But there are some problems still unsolved, such as:

1) Why doesn't moon rotate itself?

2) Why is the angle between the axis of the earth and the plane of the zodiac 23.5 degrees?

3) Why is the orbit of the revolution of the earth elliptical?

4) The sun is a focal point of the earth's orbital path, then how does the other focal point form?

5) What is the orbit of the sun? Why?

6) In the solar system, all planets have different angles between the axis of the planets and the plane of the zodiac, why?

7) Why are planets spherical?

8) What is the period of the motion of the sun?

9) Does the Newton's law have limitations?

10) What is the regularity of the changes in the earth's environment?

11) Does the universal gravitation exist? Does it have limitations? And so on. 
The second Section puts forward the methodology of this paper, which is theoretical tool for the study of this paper. The third Section of this paper derives the conclusion that the sun is a circular motion with constant velocity, and the period of the solar cycle is 19-year. In the fourth Section of this paper, we derive the conclusion of the two special laws in the period of the solar motion. The third Section and the fourth Section will be the key basis of the sun's influence on the earth. The fifth Section is the application of the law of solar motion, which is the influence of the earth earthquake (volcano) activity, and then the effect of water resources on the earth is derived. The sixth Section is the conclusion of the law of the solar movement, and the conclusion is that Newton's first law and the law of universal gravitation can be established under certain conditions.

Here we study related issues.

\section{Methodology}

Axiom 1: The universe is made up of energy. The necessary and sufficient condition for the relative stability of the universe is uniform geometric distribution of the energy of a galaxy.

Proof: 1) The universe is made up of atoms, and the atoms are made up of atoms and electrons. Either quantum mechanics or atomic energy's opinion think. The atom represents certain energy; an atom is composed of energy, and the universe is composed of energy.

2) The greatest characteristic of energy is the characteristic of tending to equilibrium. Energy tends to be balanced with energy transfer. A galaxy is the smallest unit of energy in a single energy return point.

3) According to the balance and conductivity of the energy unit (Galaxy), the energy of the galaxy is equal to that of the others. The sufficient and necessary condition for the equal and stable energy of independent galaxies is to satisfy the uniform geometric distribution.

The energy is measured by the quality, density, temperature, and kinetic energy of the independent unit or independent group. The geometric distribution can't be observed because a lot of non luminous galaxies can't be observed.

Axiom 2: Necessary and sufficient condition that the planets are spherical is their rotation and Revolution (and rotation planes meet the orbital planes), or the planet is a satellite's satellite while it is a satellite (and the two orbital planes are intersecting).

Proof: 1) A sufficient and necessary condition for a planet to be a sphere is the force back trajectory is a point (centre of sphere).

2) The rotation and Revolution (and the rotation of the tangent plane and the orbital plane intersecting) planet's rotation regression straight line trajectory and revolution return trajectory lines intersect at a point.

3) The planet itself is a satellite and a satellite of another satellite (and two orbit planes intersect), that is, the two regression lines intersect at a point.

Axiom 3: The universe is an energy vector field. The rotational motion of the galaxy leads to the power from the south to the north (energy vector).

Proof: 1) The universe is composed of relatively independent galaxies.

2) The direction of motion of the planetary regression plane in each galaxy is counter clockwise (observed from north to south).

3) The rotational kinetic energy of the planet produces an electric field and a magnetic field, resulting from the south to the north of the force (energy vector).

Theorem 1: The necessary and sufficient condition of the earth orbit is an ellipse is the movement of the sun is at a constant speed and the sun is at the two focal points with one year apart.

Proof: We assume that the sun is stationary in the revolution plane of the earth.

The movement of the earth plane and the known trajectory of the earth revolve around the sun in only one planet there, the sun.

The earth revolves around the sun's motion is relatively stable.

The trajectory of earth revolves around the sun is round. This is in contradiction with the fact that revolution of the earth orbit is elliptical.

According to the apagoge, the sun does not stop in the revolution of the earth plane, but in movement.

According to the motion of the earth revolves around the sun is relatively stable, we can obtain the sun is moving at a constant speed.

Let the focal points of the orbit of earth are F1 and F2. 
If the sun is at one focal point, it will be at another focal point one year later. Within one year of the sun moving distance is the distance between the two focal points F1 and F2. The proposition is true.

Theorem 2: The motion trajectory of the sun is the base of the cone, whose vertex is the Polaris.

Proof: According to Axiom 3, the vertical line passing through the center of the return plane revolution of the planets of the solar system also passes through the Polaris.

According to Theorem 1, the sun is moving at a constant speed.

According to the conclusion of the Section 3, the sun does the periodic movement.

The distance from the sun to the North Pole is 323 light years, and the average distance between the earth and the sun is 18 minutes and 8 seconds.

Thus the proposition is true.

Theorem 3: The necessary and sufficient condition of the moon, the earth and the sun's periodic movement is stable is the three periods are in the positive integer multiple relationships. They have the least common multiple.

Proof: Define the physical quantity — variable force impulse: real time variable size and direction of force vector and time product called variable force impulse.

Set the time period of the moon, the earth and the sun is T1, T2 and T3.

Set the variable force impulse of the moon, the earth and the sun is P1, P2, P3. The force vector of the moon, the earth and the sun is F1, F2 and F3.

Set the closed orbit of the moon, the earth and the sun is L1, L2 and L3.

Set the area of the moon, the earth and the sun's closed orbit is called S1, S2 and S3.

Set time variable $t$, we divide the time interval $[0, \mathrm{~T} 1]$ arbitrarily into $n$ small intervals, $\left[0, t_{1}\right],\left[t_{1}, t_{2}\right], \cdots$, $\left[t_{i-1}, t_{i}\right], \cdots,\left[t_{n-1}, t_{n}\right]$, where $t_{n}=T_{1}$. Take a pot $\xi_{i}(1,2, \cdots, n)$ in each $\left[t_{i-1}, t_{i}\right]$. The force $f\left(\xi_{i}\right)$ at the point $\xi_{i}$ is used as the approximate value of the interval $\left[t_{i-1}, t_{i}\right]$, and the approximate value of the variable force impulse is $f\left(\xi_{i}\right)\left(t_{i}-t_{i-1}\right)$. So the approximate value of the variable force impulse from 0 to $\mathrm{T} 1$ on the trajectory of L1 is $\sum_{i=1}^{n} f\left(\xi_{i}\right) \Delta t_{i}$.

When the time interval is divided infinitely, the limit value of the length of the length of the interval is limited; it is the impulse of the change of the moon's orbit.

$$
P 1=F 1^{*} T 1=\lim _{\substack{n \rightarrow \infty \\ \max \Delta t_{i} \rightarrow 0}} \sum_{i=1}^{n} f\left(\xi_{i}\right) \Delta t_{i}=\oint_{\substack{L 1 \\ 0-T 1}} f(t) \mathrm{d} t=S 1 \text { (constant) }
$$

Similarly, the revolution of variable force impulse trajectory period on earth is $\mathrm{P} 2=\mathrm{S} 2$ (constant).

In the same way, the variable force impulse of the sun's movement track is P3 = S3 (constant).

Variable force impulse of the moon, the earth, and the sun's movement cycle is constant. If the moon, the earth and the sun's periodic movement are in the positive integer multiple relationship and if they have the least common multiple, Then there must be a stable variable force impulse so that the value of the variable force impulse of the moon, the earth and the sun in the least common multiple time is constant. That means the necessary and sufficient condition of the moon, the earth and the sun's periodic movement is stable is the three periods are in the positive integer multiple relationships. They have the least common multiple.

\section{The Motion Law of the Sun}

Definition of energy field: The universe is made up of energy. The necessary and sufficient condition for the relative stability of the universe is uniform geometric distribution of the energy of a galaxy, the rotation of the planets generate electric energy, magnetic energy and nuclear energy. This field derived from the relatively independent group of planets is called the field of energy.

Lunar New Year's Day: January 1st with respond to the unit of the lunar periodicity.

The moon is a satellite of the earth. The earth revolves around the sun. Then solar motion is also a periodic movement. Or we can say the solar motion and the solar system's eight planets motion have periodicity.

According to Theorem 3, we will calculate the motion periodicity of the sun next.

There are many books such as China calendar, such as Reference 1 . They included the control data of hundreds or thousands of years with the lunar calendar. In this paper, which only from 1901 to 2050150 years Lunar New Year's Day (lunar January 1st) and the statistics of the calendar control. This doesn't affect the search for the periodic law of the sun, and the data from other years have no influence on the results of the study. 
The moon travels round the earth once a month (30 days). The earth's orbital period is 365 days and 11 hours.

In Table 1, we have statistical data of Lunar New Year's Days from 1901 to 2050 [1].

In order to find the law of the cycle, we establish algorithm and operate programming.

\section{Algorithm:}

1) Assign the data to an array of DATA1(r), $r=1, \cdots, 150$;

2) Loop. Loop variable from $i=2$ to 75 , Total circulation 74 times; (Description: the one-dimensional array is defined as an array of I dimensions, in which the loop variable I is equal to the number of data in each group, the data won't participate in the calculation if time is not enough);

\section{Table 1. The Lunar New Year 's Day (NYD) and AD Contrasting statistics (Month/Day).}

\begin{tabular}{|c|c|c|c|c|c|c|c|c|c|c|}
\hline Year AD & 1901 & 1902 & 1903 & 1904 & 1905 & 1906 & 1907 & 1908 & 1909 & 1910 \\
\hline Lunar NYD & $2 / 19$ & $2 / 08$ & $1 / 29$ & $2 / 16$ & $2 / 4$ & $1 / 25$ & $2 / 13$ & $2 / 2$ & $1 / 22$ & $2 / 10$ \\
\hline Year AD & 1911 & 1912 & 1913 & 1914 & 1915 & 1916 & 1917 & 1918 & 1919 & 1920 \\
\hline Lunar NYD & $1 / 30$ & $2 / 18$ & $2 / 6$ & $1 / 26$ & $2 / 14$ & $2 / 3$ & $1 / 23$ & $2 / 11$ & $2 / 1$ & $2 / 20$ \\
\hline Year AD & 1921 & 1922 & 1923 & 1924 & 1925 & 1926 & 1927 & 1928 & 1929 & 1930 \\
\hline Lunar NYD & $2 / 8$ & $1 / 28$ & $2 / 15$ & $2 / 5$ & $1 / 24$ & $2 / 13$ & $2 / 2$ & $1 / 23$ & $2 / 10$ & $1 / 30$ \\
\hline Year AD & 1931 & 1932 & 1933 & 1934 & 1935 & 1936 & 1937 & 1938 & 1939 & 1940 \\
\hline Lunar NYD & $2 / 17$ & $2 / 6$ & $1 / 26$ & $2 / 14$ & $2 / 4$ & $1 / 24$ & $2 / 13$ & $1 / 31$ & $2 / 19$ & $2 / 8$ \\
\hline Year AD & 1941 & 1942 & 1943 & 1944 & 1945 & 1946 & 1947 & 1948 & 1949 & 1950 \\
\hline Lunar NYD & $1 / 27$ & $2 / 15$ & $2 / 5$ & $1 / 25$ & $2 / 13$ & $2 / 2$ & $1 / 22$ & $2 / 10$ & $1 / 29$ & $2 / 17$ \\
\hline Year AD & 1951 & 1952 & 1953 & 1954 & 1955 & 1956 & 1957 & 1958 & 1959 & 1960 \\
\hline Lunar NYD & $2 / 6$ & $1 / 27$ & $2 / 13$ & $2 / 3$ & $1 / 24$ & $2 / 12$ & $1 / 31$ & $2 / 17$ & $2 / 6$ & $1 / 28$ \\
\hline Year AD & 1961 & 1962 & 1963 & 1964 & 1965 & 1966 & 1967 & 1968 & 1969 & 1970 \\
\hline Lunar NYD & $2 / 15$ & $2 / 5$ & $1 / 25$ & $2 / 13$ & $2 / 2$ & $1 / 21$ & $2 / 9$ & $1 / 30$ & $2 / 17$ & $2 / 6$ \\
\hline Year AD & 1971 & 1972 & 1973 & 1974 & 1975 & 1976 & 1977 & 1978 & 1979 & 1980 \\
\hline Lunar NYD & $1 / 27$ & $2 / 15$ & $2 / 3$ & $1 / 23$ & $2 / 11$ & $1 / 31$ & $2 / 18$ & $2 / 7$ & $1 / 28$ & $2 / 16$ \\
\hline Year AD & 1981 & 1982 & 1983 & 1984 & 1985 & 1986 & 1987 & 1988 & 1989 & 1990 \\
\hline Lunar NYD & $2 / 5$ & $1 / 25$ & $2 / 13$ & $2 / 2$ & $2 / 20$ & $2 / 9$ & $1 / 29$ & $2 / 17$ & $2 / 6$ & $1 / 27$ \\
\hline Year AD & 1991 & 1992 & 1993 & 1994 & 1995 & 1996 & 1997 & 1998 & 1999 & 2000 \\
\hline Lunar NYD & $2 / 15$ & $2 / 4$ & $1 / 23$ & $2 / 10$ & $1 / 31$ & $2 / 19$ & $2 / 7$ & $1 / 28$ & $2 / 16$ & $2 / 5$ \\
\hline Year AD & 2001 & 2002 & 2003 & 2004 & 2005 & 2006 & 2007 & 2008 & 2009 & 2010 \\
\hline Lunar NYD & $1 / 24$ & $2 / 12$ & $2 / 1$ & $1 / 22$ & $2 / 9$ & $1 / 29$ & $2 / 18$ & $2 / 7$ & $1 / 26$ & $2 / 14$ \\
\hline Year AD & 2011 & 2012 & 2013 & 2014 & 2015 & 2016 & 2017 & 2018 & 2019 & 2020 \\
\hline Lunar NYD & $2 / 3$ & $1 / 23$ & $2 / 10$ & $1 / 31$ & $2 / 19$ & $2 / 8$ & $1 / 28$ & $2 / 16$ & $2 / 5$ & $1 / 25$ \\
\hline Year AD & 2021 & 2022 & 2023 & 2024 & 2025 & 2026 & 2027 & 2028 & 2029 & 2030 \\
\hline Lunar NYD & $2 / 12$ & $2 / 1$ & $1 / 22$ & $2 / 10$ & $1 / 29$ & $2 / 17$ & $2 / 6$ & $1 / 26$ & $2 / 13$ & $2 / 3$ \\
\hline Year AD & 2031 & 2032 & 2033 & 2034 & 2035 & 2036 & 2037 & 2038 & 2039 & 2040 \\
\hline Lunar NYD & $1 / 23$ & $2 / 11$ & $1 / 31$ & $2 / 19$ & $2 / 8$ & $1 / 28$ & $2 / 15$ & $2 / 4$ & $1 / 24$ & $2 / 12$ \\
\hline Year AD & 2041 & 2042 & 2043 & 2044 & 2045 & 2046 & 2047 & 2048 & 2049 & 2050 \\
\hline Lunar NYD & $2 / 1$ & $1 / 22$ & $2 / 10$ & $1 / 30$ & $2 / 17$ & $2 / 6$ & $1 / 26$ & $2 / 14$ & $2 / 2$ & $1 / 23$ \\
\hline
\end{tabular}


3) DATA1(R)—DATAI (A1, , AI);

4) Let the average number of the A1 column is equal to a1, and the average of the Ai column is equal to ai;

5) Loop. Loop variable from $j=1$ to the number of rows in the array DATAI;

6) The data of the first $j$ rows of the array are subtracted (A1, $\cdots, \mathrm{AI})$ of the corresponding data, and choose the absolute value, if the difference between 28 and 32, and then reduced by 30. Because there may be a difference between the lunar cycle;

7) When the difference is greater than 2 , then $j=j+1$ returns (5); when the loop variable $J$ is equal to the maximum number of rows in the array DATAI returns (9); (Noting: New Year's day on behalf of the Chinese New Year is a time, not a day. Because Sirius added one extra day every four years, the error cannot be greater than 2);

8) Print out $i$;

9) If $I=i+1$, returns (2);

10) End, print out "exit".

Through simple programming calculation, it can be concluded that the 150 years cycle of the Lunar New Year's Day is 19 years, 38 years and 57 years. Lunar cycle and revolution of the earth, the relationship between cycle and cycle of solar motion is integer times cycle; time cycle is $19{ }^{*} \mathrm{n}$ years $(\mathrm{n}=$ natural number). That is, the minimum movement period between the sun and the other planets in the solar system is 19 years.

It can be known that the change of the earth's environment has 19-year periodicity.

\section{Periodic Law of the Change of the Earth's Environment}

In Table 2, from 1901 to 2050, we divide the Lunar New Year's Day clustering data as the same periodicity into 19 groups.

Differences between adjacent groups are $11,10,18,10,10,18,11,10,18,10,19,11,10,18,11,10,18,11$ and 19. We find that difference between the Group 19 and the Group 1 is most special and is a group of the latest New Year's Day, and followed by the Group 11 and the Group 12. It shows as Figure 1.

We conclude that the most obvious year of climate change is the Group 1, namely the year of 1920, 1939, $1958,1977,1996,2015$ and 2034. The second is Group 12, namely the year of 1912, 1931, 1950, 1969, 2008, 2026 and 2045. Obviously, the years of obviously climate change and their adjacent years are the most serious natural disasters years.

\section{The Relationship between the Energy Field and the Water Resources Cycle of the Earth}

The function of water is the buffering and neutralizing energy. The mobility of water has a great impact on the Environment.

Axiom: Under the action of the energy field, the Earth passes through the earthquake motion to tend to the Sphere [2]. Seismic fissure connected mantle and when the earth's surface is sprayed out, it turns into a volcano [3]. When the seismic gap is connected to the bottom of the sea, fresh water is produced by the decomposition And synthesis of seawater, which cooling outflow through the high mountain crate. That is the source of rivers. Or it only rises to the level of groundwater balance because of the surface pressure.

For example, when China's Qinghai Tibet Plateau formed, the first sea floor connected port is the Qaidam Basin. As the sea level felled, the basin was programmed on the ground, the second sea floor connected port became the Tarim Basin. The third the bottom of the ocean floor is the Sichuan basin and so on. At present, the Peru Chile Basin, North American Basin, the Puerto Rico Trench, Caribbean, Gulf of Mexico, Mediterranean sea, Japan Trench, Philippines Basin, Somali Basin are all undersea volcanic connected port.

After knowing the laws of motion of the sun in the field of energy, the earth's energy's North property and East property is not difficult to understand. Hurricane track in North America and the moving track of the Pacific typhoon are easier to understand.

China's haze and dust storms phenomena are through the economic development of the earth's center of gravity shift, resulting in increasing the density and more absorption of particles in the atmosphere and not other reasons. Whether willing or not, the eastward movement of the capital is inevitable.

The risk of environmental change is not a change in temperature but the change in the distribution of fresh water, including river break, groundwater pollution and loss. The development of human economy accelerates 
Table 2. The Lunar New Year's Day (1901-2050) is divided into 19 groups of the same cycle data and the difference between the groups (Month/Day).

\begin{tabular}{|c|c|c|c|c|c|c|}
\hline \multirow{2}{*}{ Group 1} & $1901(2 / 19)$ & $1920(2 / 20)$ & $1939(2 / 19)$ & $1958(2 / 17)$ & $1977(2 / 18)$ & $1996(2 / 19)$ \\
\hline & $2015(2 / 19)$ & $2034(2 / 19)$ & \multicolumn{4}{|c|}{ Difference between the next group: 11 days } \\
\hline \multirow{2}{*}{ Group 2} & $1902(2 / 8)$ & $1921(2 / 8)$ & $1940(2 / 8)$ & $1959(2 / 8)$ & $1978(2 / 7)$ & $1997(2 / 7)$ \\
\hline & $2016(2 / 8)$ & $2035(2 / 8)$ & \multicolumn{4}{|c|}{ Difference between the next group: 10 days } \\
\hline \multirow{2}{*}{ Group 3} & $1903(1 / 29)$ & $1922(1 / 28)$ & $1941(1 / 27)$ & $1960(1 / 28)$ & $1979(1 / 28)$ & 1998(1/28) \\
\hline & $2017(1 / 28)$ & $2036(1 / 28)$ & \multicolumn{4}{|c|}{ Difference between the next group: 18 days } \\
\hline \multirow{2}{*}{ Group 4} & $1904(2 / 16)$ & $1923(2 / 15)$ & $1942(2 / 15)$ & $1961(2 / 15)$ & $1980(2 / 16)$ & $1999(2 / 16)$ \\
\hline & $2018(2 / 16)$ & $2037(2 / 15)$ & \multicolumn{4}{|c|}{ Difference between the next group: 10 days } \\
\hline \multirow{2}{*}{ Group 5} & $1905(2 / 4)$ & $1924(2 / 5)$ & $1943(2 / 5)$ & $1962(2 / 5)$ & $1981(2 / 5)$ & $2000(2 / 5)$ \\
\hline & $2019(2 / 5)$ & $2038(2 / 4)$ & \multicolumn{4}{|c|}{ Difference between the next group: 10 days } \\
\hline \multirow{2}{*}{ Group 6} & $1906(1 / 25)$ & $1925(1 / 24)$ & $1944(1 / 25)$ & $1963(1 / 25)$ & $1982(1 / 25)$ & $2001(1 / 24)$ \\
\hline & $2020(1 / 25)$ & $2039(1 / 24)$ & \multicolumn{4}{|c|}{ Difference between the next group: 18 days } \\
\hline \multirow{2}{*}{ Group 7} & $1907(2 / 13)$ & $1926(2 / 13)$ & $1945(2 / 13)$ & $1964(2 / 13)$ & $1983(2 / 13)$ & $2002(2 / 12)$ \\
\hline & $2021(2 / 12)$ & $2040(2 / 12)$ & \multicolumn{4}{|c|}{ Difference between the next group: 11 days } \\
\hline \multirow{2}{*}{ Group 8} & $1908(2 / 2)$ & $1927(2 / 2)$ & $1946(2 / 2)$ & $1965(2 / 2)$ & $1984(2 / 2)$ & $2003(2 / 1)$ \\
\hline & $2022(2 / 1)$ & $2041(2 / 1)$ & \multicolumn{4}{|c|}{ Difference between the next group: 10 days } \\
\hline \multirow{2}{*}{ Group 9} & $1909(1 / 22)$ & $1928(1 / 23)$ & $1947(1 / 22)$ & $1966(1 / 21)$ & $1985(2 / 20)$ & $2004(1 / 22)$ \\
\hline & $2023(1 / 22)$ & $2042(1 / 22)$ & \multicolumn{4}{|c|}{ Difference between the next group: 18 days } \\
\hline \multirow{2}{*}{ Group 10} & $1910(2 / 10)$ & $1929(2 / 10)$ & $1948(2 / 10)$ & $1967(2 / 9)$ & $1986(2 / 9)$ & $2005(2 / 9)$ \\
\hline & $2024(2 / 10)$ & $2043(2 / 10)$ & \multicolumn{4}{|c|}{ Difference between the next group: 10 days } \\
\hline \multirow{2}{*}{ Group 11} & 1911(1/30) & $1930(1 / 30)$ & 1949(1/29) & $1968(1 / 30)$ & $1987(1 / 29)$ & $2006(1 / 29)$ \\
\hline & $2025(1 / 29)$ & $2044(1 / 30)$ & \multicolumn{4}{|c|}{ Difference between the next group: 18 days } \\
\hline \multirow{2}{*}{ Group 12} & 1912(2/18) & $1931(2 / 17)$ & $1950(2 / 17)$ & $1969(2 / 17)$ & $1988(2 / 17)$ & $2007(2 / 18)$ \\
\hline & $2026(2 / 17)$ & $2045(2 / 17)$ & \multicolumn{4}{|c|}{ Difference between the next group: 11 days } \\
\hline \multirow{2}{*}{ Group 13} & $1913(2 / 6)$ & $1932(2 / 6)$ & $1951(2 / 6)$ & $1970(2 / 6)$ & $1989(2 / 6)$ & $2008(2 / 7)$ \\
\hline & $2027(2 / 6)$ & $2046(2 / 6)$ & \multicolumn{4}{|c|}{ Difference between the next group: 10 days } \\
\hline \multirow{2}{*}{ Group 14} & $1914(1 / 26)$ & $1933(1 / 26)$ & $1952(1 / 27)$ & $1971(1 / 27)$ & $1990(1 / 27)$ & $2009(1 / 26)$ \\
\hline & $2028(1 / 27)$ & $2047(1 / 26)$ & & erence betwee & ext group: 18 & \\
\hline Groun 15 & $1915(2 / 14)$ & $1934(2 / 14)$ & $1953(2 / 13)$ & $1972(2 / 15)$ & $1991(2 / 15)$ & $2010(2 / 14)$ \\
\hline & $2029(2 / 13)$ & $2048(2 / 14)$ & & erence betwee & ext group: 11 & \\
\hline & 1916(2/3) & $1935(2 / 4)$ & 1954(2/3) & $1973(2 / 3)$ & $1992(2 / 4)$ & 2011(2/3) \\
\hline 约 & $2030(2 / 3)$ & $2049(2 / 2)$ & & erence betwee & ext group: 10 & \\
\hline$C$ & $1917(1 / 23)$ & $1936(1 / 24)$ & $1955(1 / 24)$ & $1974(1 / 23)$ & $1993(1 / 23)$ & $2012(1 / 23)$ \\
\hline & $2031(1 / 23)$ & $2050(1 / 23)$ & & erence betwee & ext group: 18 & \\
\hline Groun 18 & $1918(2 / 11)$ & $1937(2 / 13)$ & $1956(2 / 12)$ & $1975(2 / 11)$ & $1994(2 / 10)$ & $2013(2 / 10)$ \\
\hline & $2032(2 / 11)$ & & & erence betwee & ext group: 11 & \\
\hline & $1919(2 / 1)$ & $1938(1 / 31)$ & $1957(1 / 31)$ & $1976(1 / 31)$ & $1995(1 / 31)$ & 2014(1/31) \\
\hline & $2033(1 / 31)$ & & & erence betwee & ext group: 19 & \\
\hline
\end{tabular}

Noting: The error sources of a day or so in the AD calendar. Every four years we will add a day. But the mathematical expectation of every 19 year period's error is equal to zero. 


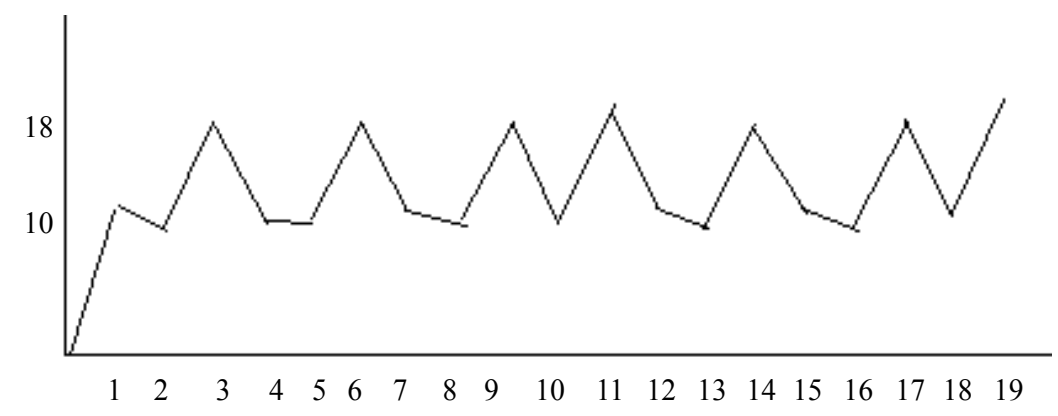

Figure 1. Inter group variation diagram (group and days).

the process of the development of the high density of atoms in the natural world. But it has greatly reduced the human survival time. There is no possibility of environmental protection for human being. There is only the possibility of reducing the environmental damage for human being. Human's mission is to accelerate the carbonation and process of hydrogen, oxygen, oxide, and fresh water (one step in the development of the height of the atomic weight of the natural world) [2]. For human beings, the best way to deal with Environmental change is to carry out rational planning of urban and rural areas first.

Space development is useless. If a planet's density is greater than the earth, the time which is suitable for human survival has passed; if a planet's density is less than the earth, the time for human survival has not yet come [2].

\section{Limit Principle of Galaxy Movement}

The universe is made up of energy, the necessary and sufficient condition for the relative stability of the universe is uniform geometric distribution of the energy of a galaxy. The ultimate direction of movement of each planet in the galaxy is the limit direction of motion.

Limit principle of galaxy movement: the movement limit of every galaxy or every planet in the galaxy is the motion toward the direction of reducing dimension regression force. It has the property that the energy sources of the neighboring galaxies tend to energy balance and energy transfer.

The solar system is 3-dimensional space. The direction of the solar energy field is the direction of the Polaris. According to the limit principle of movement above, the limit dimensionality reduction direction of motion of the solar system is the revolution of regression plane of the 8 planets.

There is no gravity on the earth. The regression force dimensionality reduction direction of the earth's rotation in the energy field is the direction of the self rotating axis. The regression force dimensionality reduction direction of revolution of the earth is the orbital plane and the line which runs through the center of the earth and is perpendicular to the orbital plane.

So it can be known that Newton's law of universal gravitation [4] holds only between two particles in the same field and in the same plane. Newton's first law [5] holds only when the objects are in the same energy field and in the same dimensionality reduction direction.

\section{Conclusion}

In this paper, through the study of the law of solar motion, we derive the conclusion that the sun is a circular motion with uniform velocity and the minimum period of the solar cycle is 19 years. We derive the solar activity cycle with two particular regularity of the conclusion, and put forward the movement of the sun, the influence of the earth earthquake (volcano) activity, and then the effect of water resources on the earth is derived. Finally, it is concluded that the first law of Newton and the law of universal gravitation can be established under certain conditions, and also we point out the broad new research prospects. We believe that the generalized gravitational force beyond a single galaxy should be proportional to the mass, density and temperature, and inversely proportional to the cube of the relative distance (we also believe that the quality, density (atomic weight) and temperature are the three factors to measure the size of energy). Of course, the reducing dimension of the central acceleration is a parameter to describe the density. This is a subject to be studied in the future. We believe the conclusion of this paper has a certain reference value for the related research. 


\section{References}

[1] Bai, H.-M. (2005) Ze Ri Tong Sheng Calendar. Hong Kong Heming Culture Press, Hong Kong, ISBN: 9789628710294.

[2] Yin, Z., Guo, Y.F. and Lai, M.S. (2011) Analysis of Climatic Change from Energy Perspective. Procedia Environmental Science, 11, 245-249.

[3] Yin, Z. (2010) The Co-Planarity and Symmetry Principle of Earthquake Occurrence. International Journal of Geosciences, 1, 38-43.

[4] Newton (1687) Newton Law of Universal Gravitation. Mathematical Principles of Natural Philosophy.

[5] Newton (1687) The First Law of Newton. Mathematical Principles of Natural Philosophy. 